\author{
C. CLEMENTE DE ARRIBA** \\ C. ECHAVARRI* \\ L. GÓMEZ PELLICO** \\ A. LÓPEZ ALONSO** \\ R. FERNÁNDEZ-VALENCIA**
}

* Servicio de Rehabilitación. Iospital Ntra. Sra. del Prado.

Talavera de la Reina, Toledo.

** Departamento de Ciencias Morfológicas

y Cirugía (Dr. Gómez Pellico).

Facultad de Medicina.

Alcalá de Henares, Madrid.

\section{Reacción ósea a implantes mixtos cerámico-plásticos. Estudio experimental preliminar}

\author{
Osseous reaction to mixed \\ ceramic-plastic implants. \\ Preliminary experimental study
}

\section{Resumen}

Se estudia la respuesta ósea a un material formado por una cerámica sintética y dos polímeros asociados. Se obtiene con ello un cilindro sólido que se coloca en la cavidad medular del femur de conejo durante distintos periodos de tiempo. Se realiza un estudio preliminar utilizando un sistema que permite obtener, en la misma preparación histológica, el implante y el tejido óseo sin descalcificar y sin tener que separarlos previamente, lo que permite un mejor estudio de la interfaz hueso-implante. Se concluye que la técnica histológica utilizada es adecuada para la valoración de la interfaz entre el hueso y el tipo de implante estudiado, igualmente dicho material resulta bien tolerado, dadas sus características de dureza, elasticidad y biocompatibilidad, aunque presenta como principal inconveniente el soportar mal las cargas mecánicas.

Palabras clave: Histología de la interfaz. Biomateriales. Técnicas histológicas.

\section{Summary}

Bone response to a material consisting of synthetic ceramic and two associated polymers was studied. A solid cylinder of this material was placed in the femoral medullary cavity of rabbits for different time periods. A preliminary study was made using a system that yielded the implant and non-decalcified bone tissue in the same histological preparation without previous separation, which produces a better bone-implant interface study. This histological technique was considered adequate for evaluating the interface between the bone and implant. This material was well tolerated, given its hardness, elasticity and biocompatibility properties, but its main disadvantage was poor resistance to mechanical loads.

Key words: Interface histology. Biomaterials. Histological techniques.

\section{Introducción}

A pesar de los grandes avances logrados en el campo de los materiales biocompatibles, no se ha obtenido aún el biomaterial que cumpla todas las características ideales, tanto estructurales como físicas, electroquímicas, económicas y de compatibilidad con el organismo ${ }^{1,2}$. Dentro del campo de las Ciencias de la Salud, han sido la Traumatología y la Implantología bucal las especialidades en las que los biomateriales se han utilizado con más frecuencia. Ambas re-

\section{Correspondencia:}

Dra. C. CLEMENTE DE ARRIBA. Dpto. Ciencias Morfológicas y Cirugía. Facultad de Medicina de la U.A.H. Ctra. Madrid-Barcelona, km. 33,600. 28805 Alcalá de Henares (Madrid). quieren el empleo de materiales compatibles con el tejido óseo circundante. Del mismo modo, las características físicas de los biomateriales utilizados deben ser muy específicas, debido, entre otras, a la existencia de factores mecánicos, como son las fuerzas que deben soportar y los movimientos en los que participan. La prótesis de cadera es una de las más utilizadas en Ortopedia hoy día, pero presenta como principal inconveniente el aflojamiento aséptico de la misma, lo que requiere una nueva intervención con el consiguiente perjuicio clínico y económico que ello conlleva. Los estudios encaminados a eliminar este problema requieren un conocimiento exacto de la interfaz hueso-implante, así como de las modificaciones que se producen a este nivel tanto de tipo celular, como bioquímico y mecánico ${ }^{3}$. 
Los factores mecánicos que facilitan el aflojamiento se inician con micromovimientos que más tarde se amplifican y ocasionan zonas de reabsorción ósea ${ }^{4}$. Una de las causas que contribuyen al aflojamiento del implante es la relación de elasticidad-rigidez del vástago ${ }^{5}$, por lo que el módulo de elasticidad del material empleado para el vástago y el diseño del mismo, así como la técnica quirúrgica de implantación, van a ser de capital importancia en el pronóstico del tratamiento. En un intento de solucionar estos problemas, han surgido múltiples tipos de prótesis, con variaciones tanto del material como del diseño y de su técnica quirúrgica.

Las prótesis de cadera se pueden clasificar en dos grandes grupos: Prótesis cementadas, utilizando para ello polímeros, y prótesis no cementadas, con fijación primaria, mediante anclaje a presión o «press-fit» en puntos concretos de la cavidad medular.

Los materiales empleados incluyen metales, polímeros y cerámicas. De forma general, los metales se emplean para el vástago femoral con o sin recubrimiento de hidroxiapatita, por su gran resistencia a las cargas; y los polímeros se usan para formar la cúpula acetabular, por su bajo coeficiente de fricción. Estos mismos materiales se han utilizado también para la fabricación de las llamadas "prótesis isoelásticas» ${ }^{6}$, que contienen un ánima metálica, ya que el polímero le confiere un índice de elasticidad más adecuado para su adaptación al tejido óseo.

Las cerámicas, por el contrario, presentan una gran resistencia a la deformación y una dureza mayor que los metales, pero presentan el inconveniente de ser muy frágiles, lo que las hace inadecuadas para su utilización como vástagos, ya que no son capaces por sí solas de absorber las deformaciones que se requieren durante el movimiento. Algunas cerámicas son bioactivas, es decir, son capaces de presentar una interacción positiva con el tejido óseo, formando un enlace químico con el mismo ${ }^{7}$.

Basándose en las propiedades de los polímeros (gran elasticidad) y las de las cerámicas (mayor dureza), se han desarrollado en los últimos años materiales compuestos formados por distintos tipos de cerámicas y polímeros ${ }^{8}$ en un intento de obtener un material que aúne ambas propiedades.

Sea cual sea el biomaterial utilizado, el hueso responde siempre de tres formas posibles:

1. Reacción de cuerpo extraño y rechazo de la prótesis de forma inmediata, en mayor o menor grado.

2. Formación de una cápsula fibrosa más o menos fina alrededor del implante.

3. Osteointegración, entendiendo por ello la formación de tejido óseo en contacto directo con la superficie del implante.

La osteointegración va asociada a un buen pronóstico en cuanto a la durabilidad de la prótesis, pero la formación de una cápsula fibrosa no indica necesariamente la pérdida a largo plazo del implante, ya que, si esta cápsula es fina y se mantiene así, puede permitir un buen funcionamiento durante años; por el contrario, si la cápsula fibrosa va aumentando de grosor, los micromovimientos de la prótesis se van haciendo más amplios y ocasionará el aflojamiento aséptico de la misma.

En el presente trabajo se analiza la utilidad de la técnica histológica empleada para obtener cortes de implantes no metálicos, así como la biocompatibilidad de un cilindro sólido experimental, formado por una hidroxiapatita sintética y dos polímeros ${ }^{9}$, que aúnan en un mismo elemento la elasticidad del polímero y la dureza de la hidroxiapatita.

LOS OBJETIVOS de nuestro trabajo fueron:

- Valoración de la técnica histológica para ser utilizada tanto en implantes metálicos como no metálicos.

- Utilización de un biomaterial compuesto (hidroxiapatita y metacrilato) para la valoración preliminar de la respuesta biológica mediante estudio de la interfaz hueso-implante.

- Realización de la técnica quirúrgica adecuada para su implantación en la cavidad medular de fémur de conejo.

\section{Material}

Como animales de experimentación se utilizaron tres conejos adultos de la cepa Nueva Zelanda. La piezas implantadas proceden del Departamento de Química Inorgánica de la Facultad de Farmacia de la Universidad Complutense de Madrid, consistiendo en 6 cilindros de $5 \mathrm{~mm}$ de diámetro por $25 \mathrm{~mm}$ de largo, compuesto por metacrilato de metilo (MMA), polimetacrilato de metilo (PMMA) e hidroxiapatita sintética (OHAp) ${ }^{10}$ (Fig. 1).

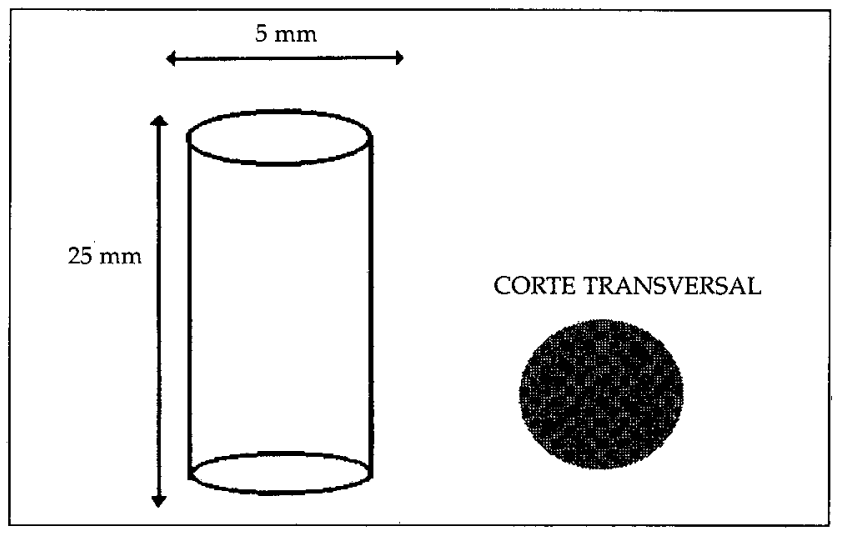

FIG. 1.-Representación gráfica del cilindro utilizado visto con lupa. Los gránulos del interior corresponden a los cristales de OHAp, el resto está formado por polímeros. 
Para el estudio histológico se utilizó el sistema de corte y pulido EXAKT que permite obtener cortes histológicos de tejidos duros sin descalcificar y del implante en conjunto.

\section{Método}

Las piezas se introducen en la cavidad medular de ambos fémures de cada conejo utilizando para ello la técnica quirúrgica que se describe a continuación:

La anestesia se afectua con una inyección intramuscular de Clorhidrato de tiazina a dosis de 0,35 $\mathrm{ml} / \mathrm{Kg}$ junto a clorhidrato de ketamina a dosis de 0,75 $\mathrm{ml} / \mathrm{kg}$ y $1 \mathrm{ml}$ de atropina. Se coloca al animal en decúbito supino, procurando que los miembros inferiores no presenten excesiva rotación, para poder facilitar las maniobras quirúrgicas.

Se practica una incisión longitudinal sobre la rótula, disecando el tejido celular subcutáneo e incidiendo longitudinalmente el periostio de la cara interna del femur, posteriormente se rechaza la rótula hacia el lado externo para visualizar los cóndilos femorales. Se realiza fresado intercondilar con broca de $6 \mathrm{~mm}$ introduciendo el cilindro experimental en el canal medular. Tras la cirugía se procede a realizar vendaje elástico que permite la deambulación espontánea.

Cada animal es sacrificado en distintos períodos de tiempo: 4, 5 y 9 semanas. Tras su sacrificio se obtienen los fémures y se fijan en formol al $10 \%$, cortándose posteriormente en fragmentos de $1 \mathrm{~cm}$ de grosor (Fig. 2).

Cada uno de los fragmentos se incluyen en metacrilato según el método de Donath y Breuner ${ }^{11}$, obteniendo de esta manera cortes de $40 \mu$ de grosor para su tinción y estudio histológico. Se utilizan las tinciones de tricrómico de Masson y picrosirius de Junqueira. Con el primero se obtiene una visión general del tejido, y el

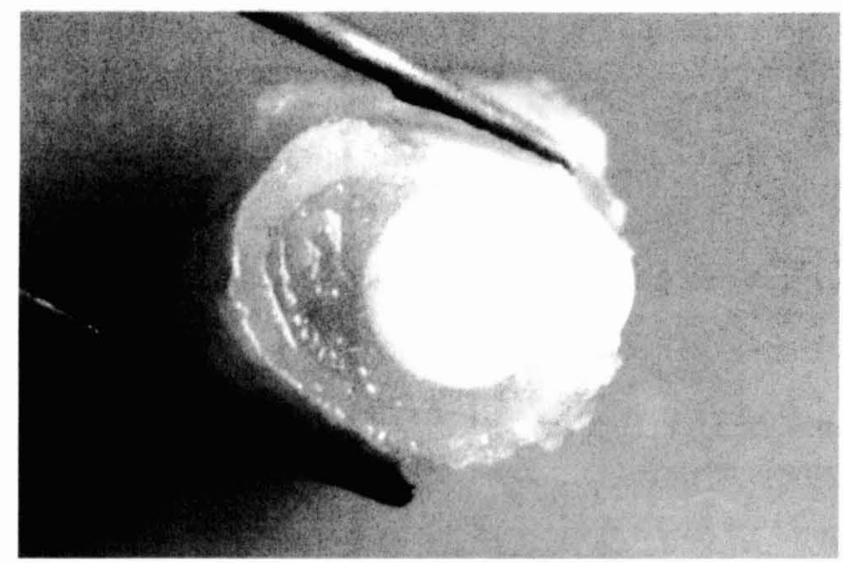

FIG. 2.-Aspecto del implante en el interior de la cavidad medular del fémur. Corte transversal. Fragmento de $1 \mathrm{~cm}$ de grosor.

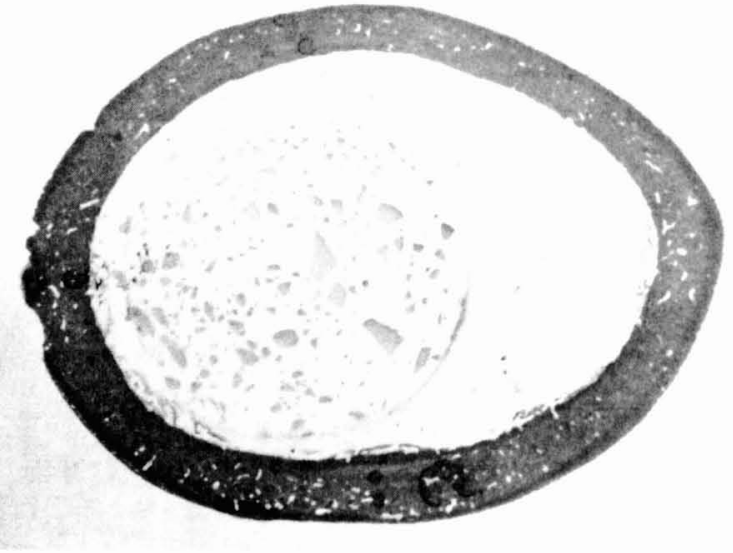

FIG. 3.-Corte transversal de la diáfisis femoral, conteniendo en la cavidad medular la sección del cilindro del implante. (En blanco el metacrilato y en rojo los cristales de OHAp). Cortical teñida en rojo, cuatro semanas de evolución. Picrosirius $7 \times$.

segundo nos permite estudiar las fibras de colágeno utilizando luz polarizada.

\section{Resultados}

Uno de los animales sufrió una fractura del tercio distal del femur izquierdo al ampliar en exceso el canal femoral. Los demás presentaron cicatrización correcta de la herida.

Los cortes transversales (Fig. 3) del hueso muestran el implante alojado en la cavidad medular que presenta un color blanco debido al polímero e inclusiones de OHAp que captan el colorante que se utilice (rojo o verde).

Hemos dividido nuestros resultados en cuatro puntos:

1. La respuesta del organismo en la superficie del implante.

2. El distinto comportamiento de la misma según el nivel de corte.

3. Variaciones de esta respuesta en el tiempo.

4. Las modificaciones que sufrió el hueso cortical.

1. Cuando el estudio histológico reveló contacto directo entre la superficie del implante y el hueso, éste adoptó, unas veces morfología de láminas paralelas $y$, otras, de láminas concéntricas formando osteonas. En otras ocasiones el implante se encontró recubierto por tejido fibroso, que con luz polarizada presentó la birrefringencia del colágeno, principal componente de este tejido.

En las zonas en las que el implante presentaba superficie rugosa, el tejido circundante, tanto óseo como conjuntivo, penetró en el interior del poro, llegando, en ocasiones, a rodear los cristales de hidroxiapatita situados en el interior del implante. 


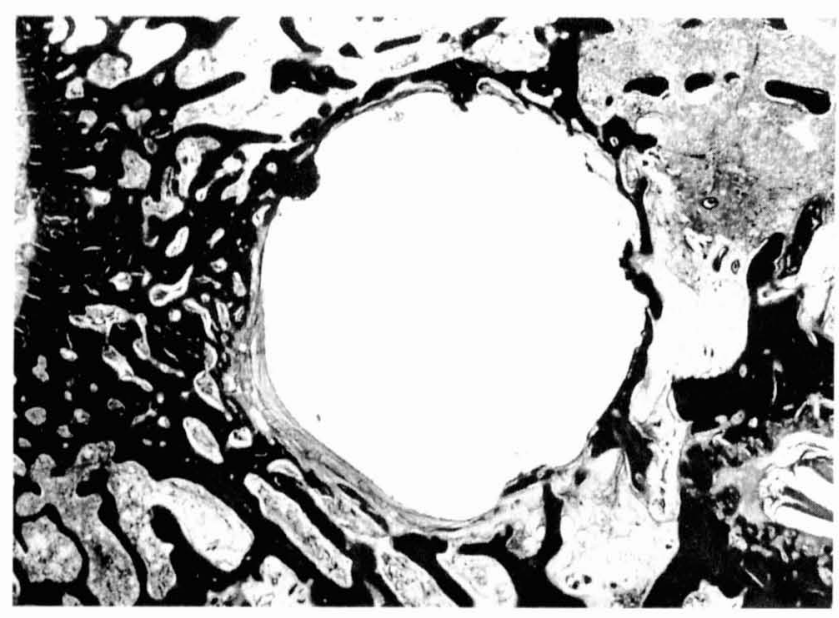

FIG. 4.-Corte transversal cercano a la rodilla en un conejo de nueve semanas de evolución. El implante está rodeado por tejido óseo en sus $2 / 3$ partes, y el resto presenta una cápsula fibrosa. Tricrómico de Masson $9 \times$.

2. Los cortes histológicos de las zonas más distales, es decir, más cercanos a la rodilla, presentaron más neoformación ósea alrededor del implante que los más proximales, donde hubo un predominio mucho mayor de tejido fibroso (Figs. 4 y 5 ).

3. Cuando se realizó el estudio de la interfaz en los distintos tiempos de evolución se encontró mayor neoformación ósea en el animal con 9 semanas de evolución, mientras que los de 4 y 5 semanas presentaron un predominio de tejido conjuntivo y tejido osteoide (Figs. 4 y 6 ).

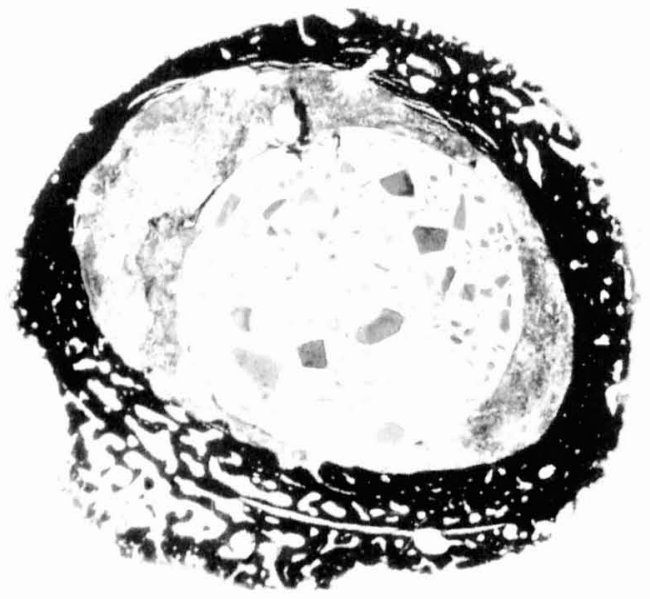

FIG. 5.-Corte en tercio medio del fémur en un conejo de nueve semanas de evolución. El implante está rodeado de una delgada capa de tejido fibroso, sin presencia de tejido óseo neoformado. Compárese con la figura 4. Tricrómico de Masson $8 \times$.

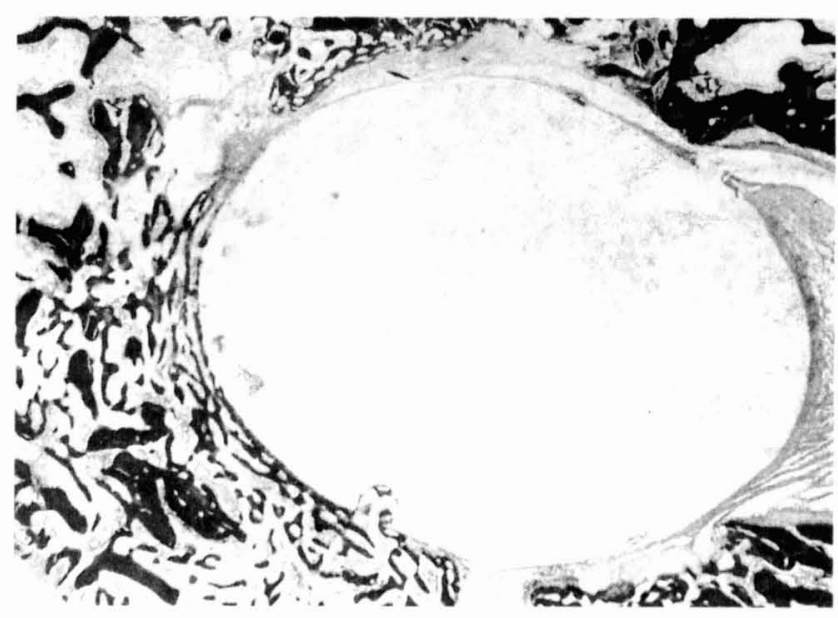

FIG. 6.-Corte transversal cercano a la rodilla, en un conejo de cuatro semanas de evolución. Nótese la menor cantidad de tejido óseo rodeando al implante, en comparación con la figura 4. Tricrómico de Masson $11 \times$.

4. La neoformación ósea que rodeó al implante se desarrolló principalmente a expensas del endostio cortical (Fig. 7). En ocasiones, la cortical del femur, en los cortes proximales, sufrió modificaciones debidas a la presencia del cilindro implantado, consistentes en una desestructuración de su morfología, con la aparición de hueso de aspecto trabecular, sustituyendo al hueso de aspecto compacto que corresponde a la cortical normal (obsérvese, el aspecto de algunas zonas de la cortical que rodea al implante en la figura 5).

5. Como último punto confirmamos la utilidad de la técnica histológica para este tipo de implantes no metálicos, ya que los cortes obtenidos fueron adecuados para su tinción y posterior estudio, utilizando los

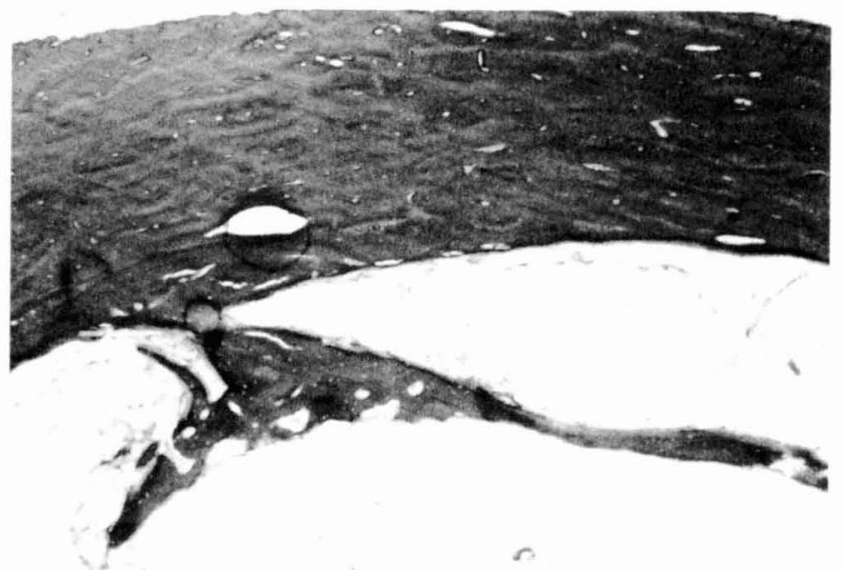

FIG. 7.-El implante está siendo rodeado por un tejido óseo que se forma a partir de la cara interna de la cortical del fémur. Tricrómico de Masson $6 \times$. 
mismos métodos que los descritos por Donath para cortes de implantes metálicos.

\section{Discusión}

Coincidimos con Geesink ${ }^{12}$ en utilizar para la técnica quirúrgica una broca algo mayor que el diámetro del cilindro a introducir, aunque ello disminuya, en parte, el anclaje a presión del implante, otros autores $^{13,14}$ utilizan brocas del mismo diámetro, pero en este caso se dificulta en exceso la introducción del implante y existe riesgo de fractura del mismo durante el proceso.

El biomaterial de estudio presentó un nivel de osteointegración aceptable, aunque nosotros no hemos cuantificado el porcentaje de osteointegración, sí parece haber una tendencia positiva a lo largo del tiempo experimental estudiado. En este sentido, otros autores ${ }^{15,16}$, encuentran porcentajes de osteointegración variables entre el $53 \%$ y el $87 \%$, utilizando titanio en perros. Nosotros encontramos formación de osteonas únicamente en el conejo de más larga evolución, y en los demás hay una delgada formación de hueso laminar. No obstante hacen falta estudios más amplios y con mayor casuística que confirmen esta tendencia.

Por otra parte, la existencia de dos respuestas distintas desde el punto de vista histológico, es decir, formación de hueso y de tejido fibroso, ha sido descrita ya por otros autores en la interfaz hueso-implante, asociándolo a una aceptable respuesta reparadora ${ }^{17,18}$, siempre que el tejido fibroso formado no sea excesivo, ya que todavía no está determinado en qué momento la excesiva producción de tejido fibroso va a empezar a provocar una pérdida de la prótesis, ni tampoco los factores que dan lugar bien a la formación de hueso o bien de tejido fibroso, aunque la cercanía del implante a la cara interna de la cortical parece ser uno de los factores que favorecería la formación de hueso, mientras que la presencia de micromovimientos del implante en el interior de la cavidad apoyaría la aparición de tejido fibroso.

Uno de los inconvenientes de las prótesis cementadas es la liberación de fragmentos sueltos de polímero, lo que ocasiona una reacción inflamatoria ${ }^{19,20}$. Horowitz y cols. ${ }^{4}$ refieren fagocitosis por macrófagos de partículas menores a $12 \mu$. Por el contrario, con el biomaterial de nuestro estudio este hecho no es posible, ya que el metacrilato forma el bloque del cilindro, no existiendo, en condiciones normales, fragmentos sueltos que puedan ser fagocitados por células inflamatorias, aunque serían necesarios estudios complementarios sometiendo a carga el implante para valorar la aparición de fragmentos sueltos.

La presencia de poros ocasionales en la superficie del implante facilita la entrada de tejido neoformado en su interior aumentando la superficie de contacto. Manley ${ }^{6}$ en 1991 describe la neoformación ósea en el interior de los poros de implantes de titanio cuando éstos están recubiertos de hidroxiapatita.

La aparición de una mayor actividad neoformadora ósea alrededor del implante, en los cortes más cercanos a la rodilla, puede deberse a que a este nivel hay hueso esponjoso cuyas trabéculas están, en algunos puntos, en íntimo contacto con el implante, y esta circunstancia podría facilitar la neoformación ósea a partir de ellas.

Es de destacar también la variación que sufrió el hueso cortical presentando un aspecto trabecular, sobre todo en los conejos de mayor tiempo de evolución. Nosotros hemos encontrado mayor trabeculación cuando el implante se encontraba cerca de la cara interna de la cortical. Linder ${ }^{2.1}$ en 1983 tras estudiar 6 casos de pérdida femoral encontró también reabsorción cortical proximal, pero no apunta ninguna causa conocida. Sin embargo otros autores, que describen igualmente osteolisis proximal, lo relacionan con las fuerzas que debe soportar la cortical ${ }^{22}$. Así, según la teoría de Schneider "en cualquier punto donde existe un fallo mecánico, o sea, en aquellas zonas donde las tensiones transmitidas son mayores que las soportadas por el hueso vivo, éste empezará a reabsorberse siendo una de las causas de aflojamiento aséptico ${ }^{23}$. Pensamos que en este modelo experimental, a pesar de que hay escasa transmisión de fuerzas verticales hacia el implante, podría haber algún tipo de carga que facilitara la remodelación ósea de la cortical, aunque también podría ser debido a otros factores relacionados con el tipo de implante $o$ con la respuesta inmune del organismo, que habrá que valorar en estudios posteriores.

\section{Conclusiones}

- El sistema de corte y pulido estudiado nos permite valorar tanto los tejidos duros orgánicos como la interfaz hueso-implante entre los distintos tipos de biomateriales (metálicos o no) que se incluyan en su interior, sin tener que realizar distintos métodos de corte histológico, por lo que los estudios comparativos serán más fáciles de realizar.

- El biomaterial experimental estudiado provoca la neoformación ósea a partir del endostio cortical.

- El hueso neoformado cubre parcialmente el implante y penetra en su superficie porosa. El resto de la superficie queda cubierta por tejido conectivo.

- La neoformación ósea es mayor en las regiones distales que en las proximales.

- El tiempo favorece la neoformación ósea, dentro del período estudiado.

- Hay desestructuración cortical en las regiones proximales, siendo más intensa cuanto mayor es el tiempo de evolución. 


\section{Agradecimientos}

Al Dr. R. Pérez Blanco, traumatólogo del Hospital Príncipe de Asturias. A los Dres Maria Vallet y Antonio Salinas y a L.M. Rodríguez de la Facultad de Farmacia de la U.C.M. por suministrar los materiales implantados. También reconocemos la ayuda económica aportada por la C.I.C. y T. a través del proyecto de investigación: MAT94-424-C2.

\section{Bibliografía}

1. Black J. Does corrosion matter? J Bone Joint Surg. 1988;70B:517-20.

2. Sunderman FW. Cobalt, chromium and nickel concentrations in body fluids of patients with porous-coated knee ir hip protheses. J Orthop Res. 1989;7(3):707-18.

3. García-Cimbrelo E. Prótesis total de cadera. Med Integral. 1993; 22(1):19-27.

4. Horowitz SM, Doty SB, Lane JM, Burstein AH. Studies of the mechanism by witch the mechanical failure of polymethylmethacrylate leads to bone resorption. J Bone Joint Surg. 1993;75-A (6):802-13.

5. Lee AJC, Ling RSM. Aflojamiento. En: Ling RSM (Eds.): Complicaciones de las artroplastias totales de cadera. Barcelona. Salvat. 1987:117-48.

6. Manley MT, Serekian P, Averill R. Preclinical histological evaluation of hydroxilapatite implants. Osteonics Corp. 1991:2-7.

7. Hench LL, Wilson J. An introduction of bioceramics. Eds: World Scientific Publishing Co. Pte. Ltd. Singapure, 1993.

8. Wuang M, Porter D, Bonfield W. Processing, characterization, and evaluation of hydroxyapatite reinforced polyethylene composites. British Ceramic Soc Trans. 1994;93(3):91-5.

9. Vallet-Regi M, Gutiérrez-Ríos MT, Alonso MP, De Frutos MI, Nicolopoulos S. Hydroxyapatite particles synthesized by pyrolysis of an aerosol. J Sol St Ch. 1994;112:58-64.
10. Vallet-Rẹgi M, Salinas A. Comunicación personal, 1996.

11. Donath $\mathrm{K}$, Breuner $\mathrm{G}$. A method for the stydy of undecalcified bones and teeth with attached soft tissues. J Oral Pathol. 1982;11: 318-26.

12. Geesink R, Groot $\mathrm{K}, \mathrm{Klein} \mathrm{C}$. Bonding of bone to apatite-coated implants. J Bone Joint Surg. 1988;70-B:17-22.

13. Pérez-Carro L, Sumillera García M, Sanz Salanova J, Donate Oliver F, Val Bernal F, Moreno Torre J. Estudio experimental de implantes de hidroxiapatita sobre titanio. Rev Ortop Traumatol. 1994;38IB(5): 423-36.

14. Soballe K, Hansen E, Rasmussen HB, Pedersen C, Bünger C. Hydroxyapatite coating enhances fixation of porous coated implants. Acta Orthop Scand. 1990;4:299.

15. Harris, $\mathrm{WH}$ : Bone ingrowth fixation of the acetabular component in canine lup joint arthroplasty. Clin Orthop, 1983, 176: 715 .

16. Hedley, AK: Bone ingrowth fixation of newly designed acetabular components in a canine model. Clin Orthop, 1983, 176: 12-18.

17. Maloney WJ, Smith RL, Castro F, Schurman DJ. Fibroblast response to metallic debris «in vitro». J Bone Joint Surg. 1993;75A:835.

18. Thomas TM, Turner M, Urgan RM, Summer DR, Galante JO. Revision, without cement of aseptically loose cement total hip prostheses. J Bone Joint Surg. 1993;75-A:845-53.

19. Agins HJ, Alcock NW, Bansal M, Salvati EA, Wilson PD, Pellicci PM, Bullough PG. Metallic wear in failed titanium-alloy total hip replacements. J Bone Joint Surg. 1988;70-A(3):34756.

20. Santavirta S, Konttinen YT, Eskola A. Immunopathological response to loose cementless acetabular components. J Bone Joint Surg. 1991;73-B(1):38-42.

21. Linder L, Lindberg L, Carlsson A. Aseptic loosening of hip prostheses. Cli Orthop Relat Res. 1983;175:93-104.

22. Rubin CT, Lanyon LE. Bone remodelling in response to applied dynamic loads. Orthop Trans. 1981;(5):237-48.

23. Remartinez Rivares JM. Estudio comparativo entre artroplastias primarias y reartroplastias con la prótesis madrepórica de Lord. Tesis Doctoral. Universidad de Alcalá de Henares, 1991 (Madrid). 